zweifellos exakter und sicherer. Ein Lyseverfahren beim Herzinfarkt bedeutet eine hohe Überlebenschance; das Medikament ist aber sehr teuer.

Im konkreten Fall befindet sich der Arzt oft im Dilemma, wenn er zu entscheiden hat, ob er diese oder jene Leistung zusätzlich erbringen soll. Nur selten kann oder will der Patient oder stellvertretend ein enger Vertrauter für das Weglassen einer auch noch möglichen Massnahme klar die Verantwortung übernehmen. Das Gegenteil lässt sich beobachten: Die Gefahr, als Arzt oder Spital Vorwürfe wegen ungenügendem Fachverhalten präsentiert zu bekommen, veranlasst oft zu übervorsichtigem Handeln. Auch auf dem Land ist die Lust, Anwälte und Patientenorganisationen einzuschalten, gewachsen.

Zunehmend stösst man auf die - zwar unausgesprochene - Meinung, am menschlichen Körper müsse doch alles jederzeit reparierbar sein. Auch ärztliche Gutachten, Haftpflichtprozesse wegen behaupteter Kunstfehler und höhere Versicherungsprämien steigern die Gesundheitskosten.

Sehr zu denken gibt mir die Entwicklung auf dem Gebiet der Invalidität. Trotz besserer Medizin nehmen die krankheitsbedingten IV-Fälle zu. Auch in der überschaubaren Belegschaft eines Landspitals ist die Zahl und der Arbeitsaufwand für pendente IV-Dossiers steigend. Gesamtschweizerisch hat die Zahl der Invaliditätsfälle in den letzten 10 Jahren auf das 1,5fache zugenommen. Zwischen 1992 und 1998 betrug die jährliche Zuwachsrate 3,7\%.

Neben den klaren somatischen Gründen sind es zunehmend psychische und nichtmedizinische Faktoren, welche dauernde Arbeitsunfähigkeiten generieren. Arbeitslosigkeit, psychosozialer Stress am und ausserhalb des Arbeitsplatzes, Burnoutsyndrom gehören zu diesen modernen Risikofaktoren, welche die Gesundheitskosten auch in die Höhe treiben.

Über allem stellt sich die berechtigte Frage, wieviel uns das Gesundheitswesen frankenmässig wert ist. Aus Umfragen und aus eigener Erfahrung wissen wir, dass für die allermeisten eine gute Gesundheit zu den höchsten Lebensgütern zählt und dass wir sehr viel einsetzen, um dieses Gut zu erhalten oder zurückzugewinnen. Gesamtschweizerisch wenden wir knapp $10 \%$ unseres Bruttosozialproduktes für das Gesundheitswesen auf (ca. 40 Mrd.), davon die Hälfte für die Spitäler. Sind denn 10\% zuviel für das höchste Gut auf dieser Erde?

\section{Eifersuchtswahn oder das Othello-Syndrom}

\author{
H. R. Gehring
}

Sie war klein, zierlich und hübsch, quicklebendig und vif, während er durchaus sympathisch, aber etwas behäbig und umständlich wirkte. Im Sprechzimmer sass er wie ein begossener Pudel neben seiner Frau und schwor, sich fortan bessern zu wollen. Das heisst, er würde ihr nicht mehr nachspionieren und augenblicklich aufhören, ihr zu unterstellen, dass sie ein Liebesverhältnis zu einem Arbeitskollegen einging, dem zehn Jahre jüngeren, attraktiven wie erfolgreichen Patrick. Sie wies den Vorwurf weit von sich: sie halte zu ihm, dem Vater ihrer Kinder und zu niemand anderem. Auch ihr Mann räumte ein, in lichten Momenten zu wissen, dass sie ihn liebe. Trotzdem überfielen ihn immer wieder Zweifel, ob sie nicht doch mit Patrick ... Dann beginne er sie zu beschatten und

Korrespondenz:

Dr. H. R. Gehring

Seminarstrasse 11

CH-3006 Bern
Leute über das Verhältnis der beiden auszufragen. «Die halbe Stadt machst du damit verrückt!» «Es ist wie ein Zwang, ein innerer Drang, gibt es da kein Mittel dagegen?»

Der Patient ist allseits orientiert und von guter Auffassung, du hättest auf Anhieb nicht vermutet, dass er als Kind Legastheniker war und während seiner ganzen Schulzeit in Kleinklassen sitzen blieb. Er litt darum an Minderwertigkeitsgefühlen. Immerhin konnte er eine Schreinerlehre und die Rekrutenschule bei der Sanität absolvieren. Mit dreiundzwanzig hatte er seine temperamentvolle und hübsche Gattin geheiratet, die seit der Geburt des 2. Kindes unterbunden ist. Bis zum Auftreten der krankhaften Eifersucht verlief ihre Ehe, nach Angaben beider Partner, sehr befriedigend, seither sei sie massiv belastet. Auch eines der Kinder habe mit Magen-Darm-Beschwerden reagiert. Die Frau lehne ihn jetzt sexuell ab, da sie sich durch sein Misstrauen zutiefst verletzt fühle, was seine Eifersucht wiederum anstachelt.

Handelt es sich um eine einfühlbare Phantasie, wie sie in jeder Beziehung vorkommen kann, aus einer depressiven Stimmung heraus geboren, wenn das Selbstwertgefühl nicht über jeden Zweifel erhaben ist, oder leidet der Mann an einem eigentlichen Eifersuchtswahn? Alkohol oder Drogen, wie man vermuten könnte, sind nicht im Spiel, was die Frau bestätigt. Halluzinationen, merkwürdige Körperempfindungen oder Entfremdungsgefühle verneint er. Allerdings finden beide, er sei nicht mehr derselbe 
wie früher. Festgemacht hatte sich seine fixe Idee, als er eines Abends seine Frau in der Quartierbeiz mit dem Arbeitskollegen ein Bier trinken und die beiden fröhlich lachen sah. Diese Eingebung wertet er stärker als alle Beteuerungen der Gemahlin und das $\mathrm{Zu}$ reden wohlmeinender Freunde.

Während du den beiden zuhörst, besinnst du dich auf die Trias von Jaspers, welche einen Wahn kennzeichnet: Subjektive Gewissheit, Unmöglichkeit und Unkorrigierbarkeit. Unmöglich ist die Beziehung einer attraktiven Frau zu einem Arbeitskollegen sicher nicht. Anders als die Behauptung, es sei jemand der Kaiser von China oder wenn er huste, gehe die Welt unter.

Auch die subjektive Gewissheit deines Patienten ist nicht absolut. Zwischendurch weiss er nämlich, dass seine Frau ihm treu ist. Also führst du die antidepressive Behandlung des Hausarztes fort und wechselst auf ein Präparat, von dem die Firma behauptet, es wirke auch gegen Zwangsideen.

Nach vorübergehender Besserung spitzte sich die unhaltbare Situation zu. Wieder quälte er seine Frau mit seinen Anschuldigungen, entfernte sich immer häufiger von der Arbeit, um "Beweise für ihre Treue» zu finden. Die Unkorrigierbarkeit dieses Wahns steht fest.

Ein merkwürdiges Gefühl beschleicht dich bei der nächsten Konsultation. Das Wort Othello spukte schon einmal durch deinen Kopf, aber du legtest es rasch beiseite. Hatte der Mohr von Venedig doch einen Liebeswahn? Mehr zufällig als gezielt legst du in der Zwischenzeit die Platte mit Verdis Oper auf, eine der späteren Einspielungen von Placido Domingo als Othello, dem Othello. Nein, du erinnerst dich wieder: er litt nicht an einem Liebes-, sondern an einem Eifersuchtswahn wie dein Patient. Fuggirmi io sol non so! Sangue! Ich allein kann mir nicht entkommen! Blut!

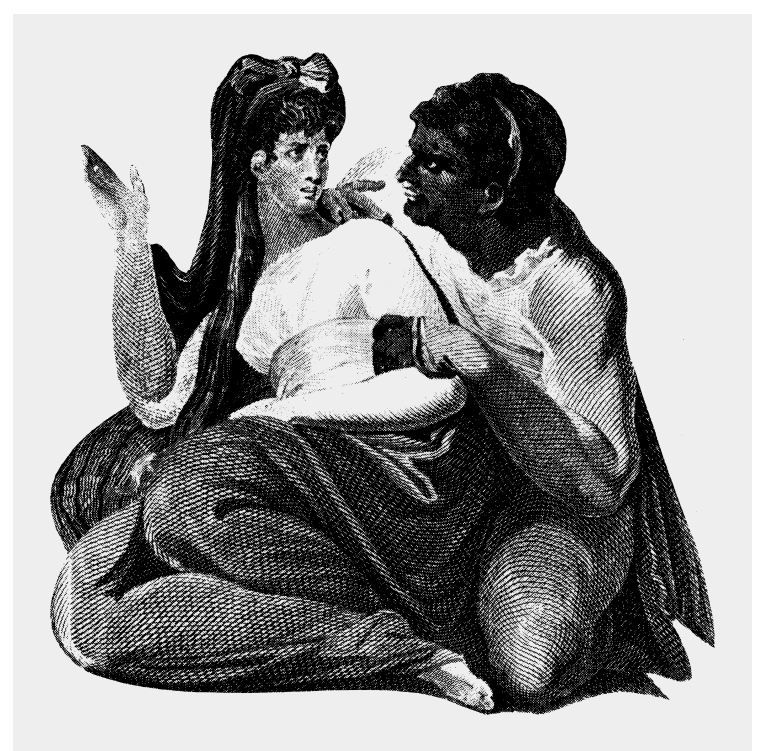

Das geht dir unter die Haut. Plötzlich wirst du inne, dass dieses Stück mit Mord und Selbstmord endet. Nicht selten bemerken Patienten, wenn in uns zwischen zwei Konsultationen etwas Wichtiges vor sich ging. Er schaut dich heute ganz anders an, flehend: "Ich habe Angst!" Fuggirmi io sol non so! Sangue! Klingt es in dir nach. "Befürchten Sie, etwas Unüberlegtes zu tun?» Er nickt: "Ich habe Angst vor mir!» Mit der Einweisung in die Klinik ist er einverstanden und später sogar erleichtert. Etwas habe er dir noch nicht sagen können, liess er dir ausrichten: dass er eine Stimme höre, die ihm von der Untreue seiner Frau erzähle. Jagos Stimme? Jenem Bösewicht, den Shakespeare schon am Anfang seines Othello sagen lässt: "I am not what I am!» Du nimmst dir vor, wieder häufiger ins Theater zu gehen. 